\title{
Performance Improvement of video based applications in WFQ using Committed Access Rate
}

\author{
Neha Singh \\ National Institute of Technology, \\ Jalandhar, India
}

\author{
Ajay K Sharma \\ National Institute of Technology, \\ Jalandhar, India
}

\begin{abstract}
In this paper, the video received, IP drop in WFQ is improved using committed access rate (CAR). The committed access rate is used for video and http applications and for WFQ queuing scheme. From results, it has been analyzed that the video received in WFQ shows much better improvement than the conventional. The significant results have been investigated for IP drop in case of WFQ with committed access rate and conventional which are 0.7 and 8.36 packets per second respectively. The result for video received in case of WFQ with committed access rate and conventional is 383.8 and 5.56 packets per seconds.
\end{abstract}

\section{Keywords}

Weighted Fair Queue, Committed Access Rate (CAR), IP QoS, ToS

\section{INTRODUCTION}

Committed access rate is a method for managing unwanted traffic on the network without affecting the important traffic. CAR set high TOS for video traffic and low TOS for HTTP traffic. WFQ can prioritize the traffic and give the high bandwidth to the video traffic. CAR provides high bandwidth for video applications. CAR defines a rate-limiting feature for policing traffic, which helps managing the network's access bandwidth policy by ensuring that traffic falling within specified rate parameters is transmitted, while dropping packets that exceed the acceptable amount of traffic or transmitting them with a different priority. The input and output traffic on an interface or sub-interface may be selected based on a flexible set of criteria like all IP traffic or all traffic on an interface or based on IP precedence or MAC address or IP Access List, after which it applies the contracted rate limits, which are the parameters for a token bucket algorithm i.e. average rate, normal burst size and excess burst size. Once the packet is determined to be conforming or exceeding its limits the defined conform/ exceed action will be taken. Committed access rate can be used with the IP traffic. It doesn't work for non-IP traffic like AppleTalk, IPX, SNA, DECNet [1].

CAR is a limiting or policing scheme which consists of certain rules based on attributes such as class of service (COS), data rate, normal burst size and excess burst size which sets a bandwidth limit for specific types of traffic on router interfaces. For example, you can create a policy that limits web traffic to 200 kilobits/sec. This puts a cap on the bandwidth available to that traffic, ensuring that the remainder of the interface's bandwidth is available to other kinds of traffic. In this example, if web traffic does not fill 200 kilobits/sec, other kinds of traffic can use the unused bandwidth. Packets are dropped if traffic bursts exceed the limit. CAR limiting does not attempt to smooth or shape the traffic flow in the way that GTS or FRTS attempt to do. Because CAR does not buffer the traffic, there is no delay in sending it, unless the traffic flow exceeds your rate policy and it is dropped. One of the main uses for limiting policies is to ensure that traffic coming into the network is not exceeding agreed-upon rates. If the limiting policy for inbound traffic is defined, the traffic can be controlled before it gets into your network at the inbound interface [2].

Traffic policing controls the maximum rate of traffic sent or received on an interface. Based on the results of the token bucket measurement, an action can be configured to mark packets and separate packets into multiple classes or levels of service. Traffic polices provide two key benefits:

Bandwidth management through rate limiting: It control the maximum rate of traffic sent or received on an interface. Traffic that falls within the rate parameters is sent, whereas traffic that exceeds the parameters is dropped, or sent with a different priority. Packet marking through IP precedence, QoS group, or DSCP value setting: Packet marking partition the network into multiple priority levels or classes of service $(\mathrm{CoS})$ [3]. CAR is used to set the IP precedence for packets entering the network. CAR controls the bandwidth of a certain type of traffic, and an access control list (ACL) defines which traffic it controls. Once the ACL is created, the CAR can be set to enforce a bandwidth rate on that traffic in either an inbound or outbound direction, according to the interface on which CAR applied. The traffic shaping or limiting (traffic policing) policies can be created on a device's interface to manage how much of the interface's bandwidth should be allocated to a specific traffic flow. The policies based on a variety of traffic characteristics, including the type of traffic, its source, its destination, and its IP precedence settings (traffic coloring) can be set accordingly. Shaping differs from limiting in that shaping attempts to smooth the traffic flow to meet the rate requirements, whereas limiting (traffic policing) does not smooth the traffic flow; it only prevents the flow from exceeding the rate. Unlike queuing techniques, which are part of an interface's characteristics, generic traffic shaping or traffic limiting is done through policies that are defined in access control lists (ACLs). Queuing techniques affect traffic only when an interface is congested, or in the case of WRED, when traffic exceeds a certain threshold. With traffic shaping policies, flows are affected even during times of little congestion.

\section{SYSTEM MODELING AND SCENARIOS}

The OPNET modeler tool is a network simulator which provides a virtual network environment that models the behavior of an entire network, including its routers, switches, protocols, servers, workstations, different kinds of links and individual applications. 


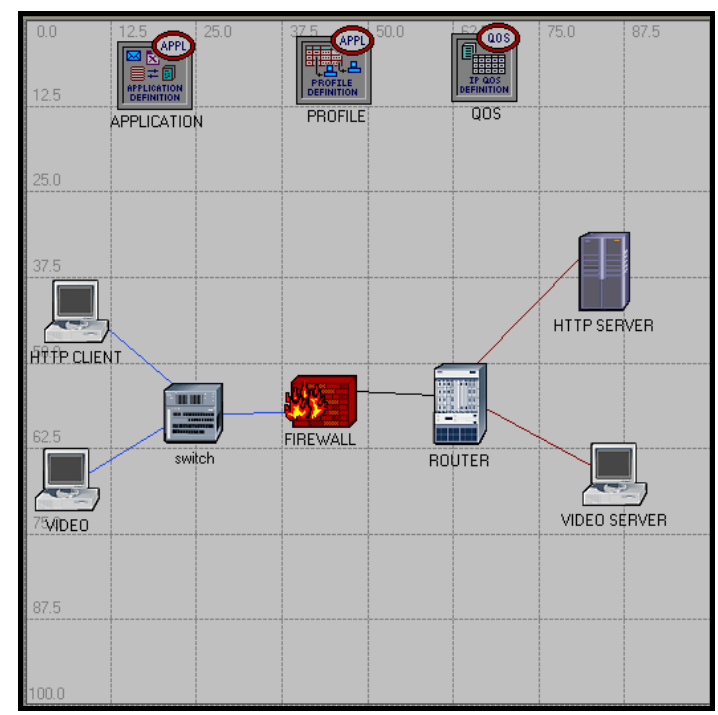

Figure 1: Simulation Model

Fig 1 shows the simulation model consisting of two domains firewall and router. The firewall consisting of 2 clients http and video applications. In this simulation model, committed access rate is enable. So those in the transmitter side token ring clients are used. Firewall router is of token ring, without token ring it can't enable the properties of CAR. Now at receiver side all the server and router are of Ethernet. Http and video clients are of token ring connected with switch via TR link and this link is connected with firewall. This firewall is connected with router via PPP DS3 link (Data rate of PPP DS3 link is $44.736 \mathrm{mbps}$ ) and the router is connected with the http server and video server via 10BaseT.

\section{RESULTS AND DISCUSSION}

In this paper, the performance of video received, IP drop is improved using committed access rate. The parameters that are taken into consideration are: IP packet drops and video receive and Delay.

Fig 2 shows the graph for IP drop in case of WFQ. The result has been investigated for CAR and default IP drop in case of WFQ is 0.7 and 8.36 packets per second respectively. It has been investigated from graph that there is more drop in default (WFQ) as compared to committed access rate.

Fig 3 depicts the graph for voice received (in case of WFQ. The results shown for CAR and default in case of WFQ is 383.8 and 5.56 packets per seconds respectively. From the simulation results it has been analyzed that the video received in WFQ is more using committed access rate.

Fig 4 illustrates the graph for delay in case of WFQ. The result has obtained for delay in case of CAR and default is $0.0005 \mathrm{~ms}$ and 2.38 seconds respectively. It has been analyzed from the simulation results that there is less delay in Car as compared to default.

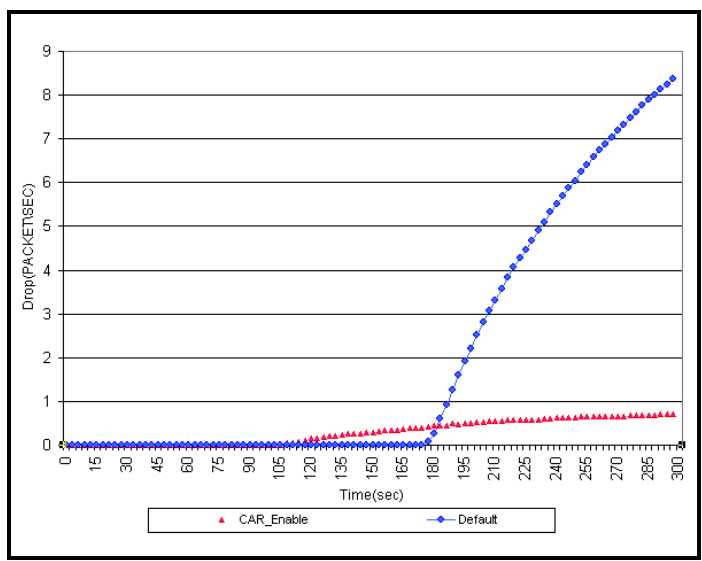

Figure 2: IP Packet Drop for WFQ

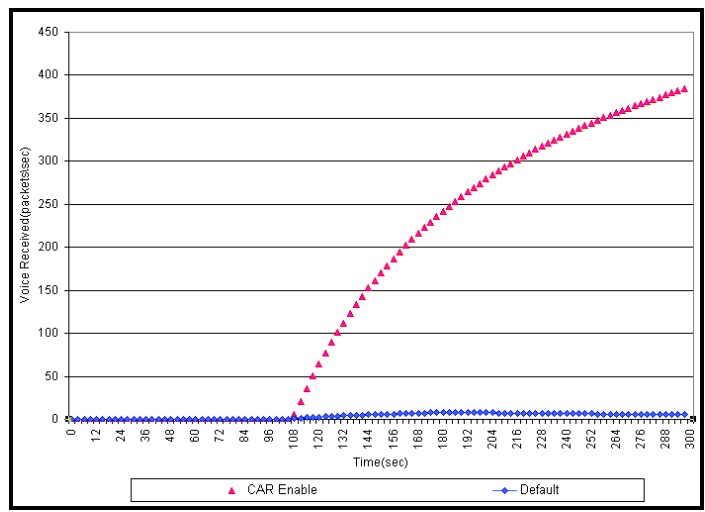

Figure 3: Video Received for WFQ

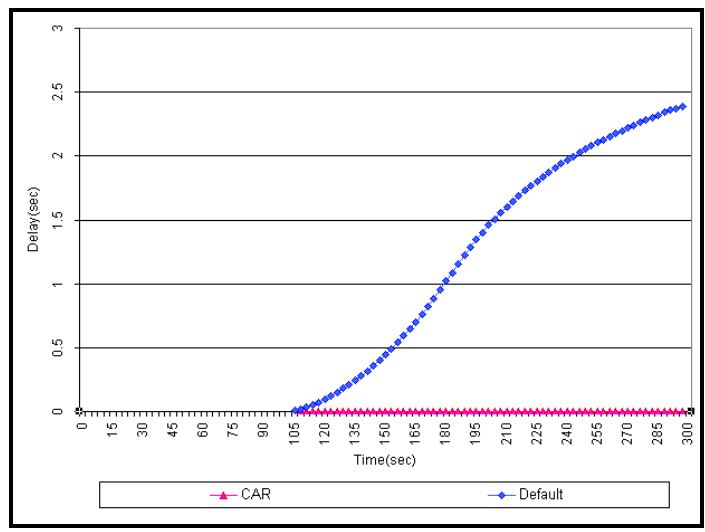

Figure 4: Delay for WFQ

\section{CONCLUSIONS}

In this paper, the performance of video received, IP drop and delay is improved using committed access rate. The simulation results are obtained in OPNET modeler 10.0. The traffic classes and prioritization allow us to encourage network applications to classify their traffic and transport only the highest-value bits during heavy congestion conditions. The result shows improvement in IP Packets drop, video receive and delay in WFQ 
using CAR as compared to conventional. So results clearly depicts that during heavy congestion, the performance of weighted Fair Queue is improved using committed access rate.

\section{REFERENCES}

[1] Configuring committed access rate "Cisco IOS Quality of Service Solutions Configuration Guide" mail. attelecom.com/security_training/qcfcar.pdf

[2] Planning for quality of service, wwwchina.cisco.com/univercd/cc/td/doc/product/rtrmgmt/ciscoas u/class/qpm1_1/using_qo/c1plan.htm, page 19

[3] Cisco "Comparing Class-Based Policing and Committed Access "Rate". http://www.cisco.com/en/US/tech/tk543/tk545/technologies_ tech_note09186a00800d7276.shtml
[4] Peter J. Welcher"Quality of Service feature", http://www.netcraftsmen.net/welcher/papers/qosoverall.html.

[5] Quality of service for voice over IP. http://www.cisco.com/en/US/docs/ios/solutions_docs/qos_so lutions/QoSVoIP/QoSVoIP.html

[6] Federico, di Napoli, Centra Servizi Didattico Scientifico, and V. Cinthia. 2003. Improving the Performance in Multimedia Streaming Network: A DIFFERENTIATED SERVICE APPROACH, Page 3-5, 9, IEEE.

[7] E.QOS: Solutions for Service Providers using Riverstone Networks' Switch Routers, white paper.

[8] Mirjana Stojanovic. 2003. An Approach for Planning and Design of QoS Enabled IP Networks, pp 1-3, IEEE.

[9] Srinivas Vegesna: "IP Quality of Service" Cisco 2003. 